\title{
Synthesis, Characterization and Biological Activities of Hydrazone Schiff Base and its Novel Metals Complexes
}

(Sintesis, Pencirian dan Aktiviti Biologi Hidrazon Pangkal Schiff serta Kompleks Baru Logamnya)

\author{
IQbal Hussain, Aaqib Ullah, AtTa Ullah Khan, Wali Ullah Khan, Riaz UllaH*, \\ AbDelaAty A. Shahat AlmoQbil A. NASER \& HAFIZ MaJid MAHMOOD
}

\begin{abstract}
Recently, researchers are focusing on the synthesis of Schiff base complexes due to their promising biological activities and frequent use in the pharmaceuticals. In the present study we synthesized Schiff base of salicyldehyde and 2, 4-dinitrophenylhydrazine by modified method and its novel metal complexes with Cu (II), Zn (II), Co (II), Ni (II), Mn (II), V (II) and AU (IIII). For spectroscopic studies and characterization, UV-Vis and FT-IR spectroscopy were used. The Schiff base and its metals complexes were tested for antimicrobial, cytotoxic and antioxidant activities. Significant results are showed by the all synthesized complexes.
\end{abstract}

Keywords: Biological activities; IR spectroscopy; Schiff base; UV-visible

ABSTRAK

Baru-baru ini penyelidik menumpukan perhatian kepada sintesis kompleks pangkal Schiff disebabkan oleh aktiviti biologi yang berpotensi dan kerap digunakan dalam bidang farmaseutis. Dalam kajian ini, Schiff pangkal salisildehid dan 2, 4-dinitrofenilhidrazin disintesis menggunakan kaedah ubah suai dan kompleks baru logamnya dengan Cu (II), Zn (II), Co (II), Ni (II), Mn (II), V (II) dan AU (IIII). Untuk kajian spektroskopi dan pencirian, UV-Vis dan Spektroskopi FT-IR telah digunakan. Pangkal Schiff dan kompleks logam telah diuji untuk aktiviti antimikrob, sitotoksik dan antioksidan. Hasil ketara telah ditunjukkan oleh semua kompleks yang telah disintesis.

Kata kunci: Aktiviti biologi; pangkal Schiff; spektroskopi IR; UV nampak

\section{INTRODUCTION}

Hydrazones are the derivatives of hydrazine and an essential group of drugs. Currently, they have been focused to, due to their wide range of pharmaceutical applications (Zahid et al. 1997). They have general formula R-NH-N=CRR", synthesized through reduction reaction of hydrazine with carbonyl compounds (Hany et al. 2015). Hydrazones are more reactive than carbonyl groups (aldehyde or ketone) due to the presence of alpha active hydrogen atoms. They are used as precursor in organic reactions (Florian et al. 2006), particularly in the preparation of Schiff base ligand (Zeyrek et al. 2006) which formed coordination bond with transition metals in vivo, with promising biological properties (Savini et al. 2002), and also used as ligands in coordination chemistry. It is reported in the literature that hydrazones complexes employed in medicines also (Hajar et al. 2010; Ochiai 1977). Their complexes with different metals showed antituberculosis (Katyal \& Dutt 1975) herbicidal, insecticidal, and nematocidal activities (Amrata et al. 2014; Li-Fei et al. 2012; Rajarajan et al. 2016). It is also reported that hydrazones Schiff base and their metal complexes with various transition metals possess anti-analgesic, antiinflammation, antibacterial, antifungal and anticancer activities (Hassan et al. 2007; Jin et al. 2006). On the basis of the cited valuable outcome and their vital role in medicine and wide applications in the pharmaceuticals, as therapeutic agents in the oxidation-reduction process in the chemical industries, in the oxidation-reduction reactions of different functional groups in organic chemists, used as insecticidal and agrochemicals in parts. The current initiative was therefore undertake, we synthesized salicyldehyde-2, 4-dinitrophenylhydrazone and its novel metals complexes $\mathrm{Cu}$ (II), Zn (II), Co (II), $\mathrm{Ni}$ (II), Mn (II), V (II) and AU (IIII).

\section{EXPERIMENTAL DETAILS}

\section{PREPARATION OF SDNPH LIGAND $\left(\mathrm{C}_{13} \mathrm{H}_{10} \mathrm{~N}_{4} \mathrm{O}_{5}\right)$}

The Schiff base was synthesized through similar procedure cited in the literature (Shahawi et al. 2013; Silverstein \& Bassler 1981) by the condensation reaction of salicyldehyde (0.414 mL, $3.9 \mathrm{mmol})$ with 2, 4-dinitrophenylhydrazine $(0.594 \mathrm{~g}, 3 \mathrm{mmol})$ in $10 \mathrm{~mL}$ of ethanol (Scheme 1). The resultant mixture was stirred for $3 \mathrm{~h}$ at $25^{\circ} \mathrm{C}$ and monitored by TLC. It gives orange color precipitate, which was filtered, rinsed with $\mathrm{EtOH}$ and dried. 


\section{PREPARATION OF METALS COMPLEXES}

All the metals complexes were synthesized by the reaction of salicyldehyde 2, 4-dinitrophenylhydrazone (SDNPH) schiff base with metals salts (in the form of sulphate and chloride). SDNPH schiff base $(0.4 \mathrm{~m} \mathrm{~mol})$ was dissolved in $3 \mathrm{~mL}$ of ethanol and was added dropwise to the $(0.2$ $\mathrm{m}$ mol) metal solution (ethanol). The reactions mixture was stirred for 5-15 h at room temperature with a small amount of $\mathrm{NaOH}$ in ethanol. Different colors precipitates were obtained which was filtered, washed with $\mathrm{EtOH}$ and dried. The Proposed Structure of M (II) and M (III) metal complexes are shown in Scheme 2.

\section{RESULTS AND DISCUSSION}

All the synthesized compounds were of different color, stable and insoluble in polar solvents but readily soluble in DMF and DMSO. They do not have sharp melting point. The physicals properties are given in the Tables 1 and 2 .

\section{INFRARED SPECTROSCOPY}

Infrared spectroscopy is a very useful method for the structure elucidation of compounds, that is ligands and their complexes to show the moiety bonded to the center metals atoms subsequently. The infrared records for the Schiff base ligand and its compounds are given in Table
3. Infrared bands of Schiff base and its complexes were weighed in the region of $4000-400 \mathrm{~cm}^{-1}$ to find out the coordination of ligand to metals ions. The difference between the ligand spectrum and its complexes showed coordination of Schiff base with metals ions. Salicyldehyde-2, 4-dinitrophenylhydrazone Schiff base show a characteristic band of $v(\mathrm{OH})$ of phenol group which reduced due to inter molecular $\mathrm{H}$-bonding, is observed at $3300-3620 \mathrm{~cm}^{-1}$ in the spectrum of ligand. In addition, followed by $3273,1616.79,1513.41,1334.55$, 1272.00 and 1085.80 regions assigned to $\mathrm{N}-\mathrm{H}, \mathrm{C}=\mathrm{N}$, $\mathrm{NO}_{2}, \mathrm{Ar}-\mathrm{O}$ and $\mathrm{N}-\mathrm{N}$ stretching vibration (Shahawi et al. 2013; Silverstein \& Bassler 1981). The vanishing of the $\mathrm{OH}$ broad peak in all coordination compound confirmed $\mathrm{M}-\mathrm{O}$ bond after complexation (Leniec et al. 2006). The absorption band at 1616.79 allocated to $\mathrm{C}=\mathrm{N}$ of Schiff base, changing in frequency on complexation with metals ion representing the coordination of imine $\mathrm{N}$ to $\mathrm{M}$ formed M-N bond (Kaczmarek et al. 2009; Misbah et al. 2013).

IR spectral shift of different compounds are comparable, representing like structures of the metals complexes. The new bands in range of complexes were observed for $v(\mathrm{M}-\mathrm{O})$ and $v(\mathrm{M}-\mathrm{N})$ stretching at region of 660-688 and 520-547 $\mathrm{cm}^{-1}$ (Guo et al. 2006) confirmed the coordination of Schiff base with metals ions. Spectral data shows that all the coordination compounds are<smiles></smiles>

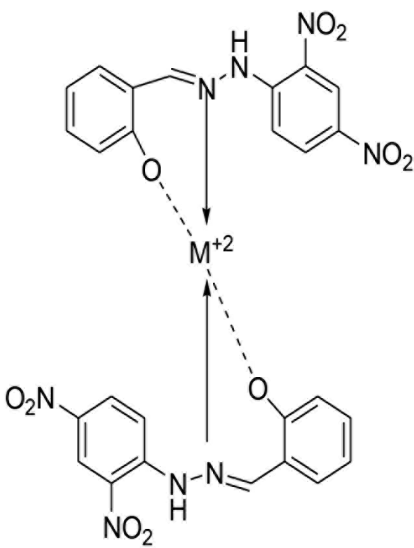

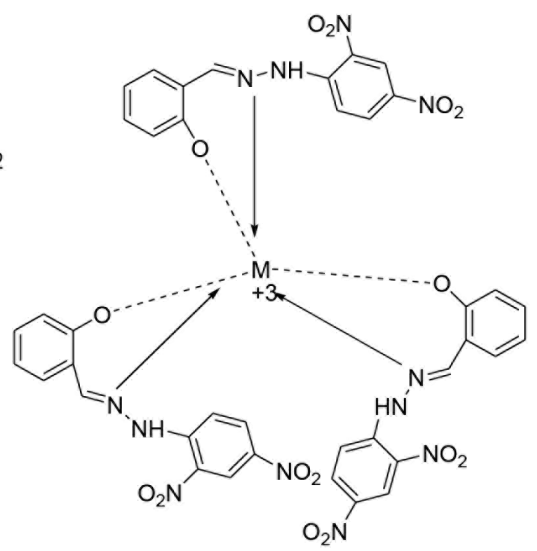

SCHEME 2. 
TABLE 1. Physicals properties of Schiff base and its complexes

\begin{tabular}{lcccc}
\hline Compounds & M. Mt & Color & M. Point & \% Yield \\
\hline $\mathrm{C}_{13} \mathrm{H}_{10} \mathrm{~N}_{4} \mathrm{O}_{5}$ & 302 & Orange & 257 & 80.09 \\
$\mathrm{Cu}\left(\mathrm{C}_{13} \mathrm{H}_{10} \mathrm{~N}_{4} \mathrm{O}_{5}\right)_{2}$ & 665.54 & Light pink & 268 & 63.05 \\
$\mathrm{Ni}\left(\mathrm{C}_{13} \mathrm{H}_{10} \mathrm{~N}_{4} \mathrm{O}_{5}\right)_{2}$ & 660.69 & Brown & $>300$ & 64.44 \\
$\mathrm{Zn}\left(\mathrm{C}_{13} \mathrm{H}_{10} \mathrm{~N}_{4} \mathrm{O}_{5}\right)_{2}$ & 667.93 & Yellowish & 287 & 65.72 \\
$\mathrm{Co}\left(\mathrm{C}_{13} \mathrm{H}_{10} \mathrm{~N}_{4} \mathrm{O}_{5}\right)_{2}$ & 660.93 & Light orange & 246 & 68.37 \\
$\mathrm{Mn}\left(\mathrm{C}_{13} \mathrm{H}_{10} \mathrm{~N}_{4} \mathrm{O}_{5}\right)_{2}$ & 656.93 & Dark pink & $>300$ & 69.30 \\
$\mathrm{Au}\left(\mathrm{C}_{13} \mathrm{H}_{10} \mathrm{~N}_{4} \mathrm{O}_{5}\right)_{3}$ & 798.97 & Brick red & 265 & 62.98 \\
$\mathrm{Cd}\left(\mathrm{C}_{13} \mathrm{H}_{10} \mathrm{~N}_{4} \mathrm{O}_{5}\right)_{2}$ & 714.41 & Red & 273 & 57.98 \\
$\mathrm{~V}\left(\mathrm{C}_{13} \mathrm{H}_{10} \mathrm{~N}_{4} \mathrm{O}_{5}\right)_{2}$ & 652.49 & Reddish orange & 260 & 55.79 \\
\hline
\end{tabular}

TABLE 2. Solubility of Schiff base and its complexes

\begin{tabular}{|c|c|c|c|c|c|c|c|c|c|}
\hline \multirow{2}{*}{ Solvents } & \multicolumn{9}{|c|}{ Complexes } \\
\hline & S.B & $\mathrm{Cu}$ & $\mathrm{Ni}$ & $\mathrm{Co}$ & $\mathrm{Zn}$ & $\mathrm{Mn}$ & $\mathrm{Au}$ & $\mathrm{Cd}$ & V \\
\hline$n$-Hexane & $\mathrm{N}$ & $\mathrm{N}$ & $\mathrm{N}$ & $\mathrm{N}$ & $\mathrm{N}$ & $\mathrm{N}$ & $\mathrm{N}$ & $\mathrm{N}$ & $\mathrm{N}$ \\
\hline Ethanol & $\mathrm{P}$ & $\mathrm{P}$ & $\mathrm{P}$ & $\mathrm{P}$ & $\mathrm{P}$ & $\mathrm{N}$ & $\mathrm{P}$ & $\mathrm{CS}$ & $\mathrm{CS}$ \\
\hline Diethyl ether & $\mathrm{P}$ & $\mathrm{P}$ & $\mathrm{P}$ & $\mathrm{P}$ & $\mathrm{P}$ & $\mathrm{P}$ & $\mathrm{P}$ & $\mathrm{P}$ & $\mathrm{CS}$ \\
\hline Chloroform & $\mathrm{CS}$ & $\mathrm{P}$ & $\mathrm{P}$ & $\mathrm{CS}$ & $\mathrm{P}$ & $\mathrm{P}$ & $\mathrm{CS}$ & $\mathrm{CS}$ & $\mathrm{CS}$ \\
\hline $\mathrm{THF}$ & $\mathrm{CS}$ & $\mathrm{CS}$ & $\mathrm{P}$ & $\mathrm{CS}$ & $\mathrm{CS}$ & $\mathrm{P}$ & $\mathrm{CS}$ & $\mathrm{CS}$ & $\mathrm{CS}$ \\
\hline Ethyl acetate & $\mathrm{CS}$ & $\mathrm{CS}$ & $\mathrm{P}$ & $\mathrm{CS}$ & $\mathrm{P}$ & $\mathrm{P}$ & $\mathrm{CS}$ & $\mathrm{CS}$ & $\mathrm{CS}$ \\
\hline Methanol & $\mathrm{CS}$ & $\mathrm{CS}$ & $\mathrm{P}$ & $\mathrm{CS}$ & $\mathrm{CS}$ & $\mathrm{P}$ & $\mathrm{CS}$ & $\mathrm{CS}$ & $\mathrm{CS}$ \\
\hline DMSO & $\mathrm{CS}$ & CS & CS & $\mathrm{CS}$ & $\mathrm{CS}$ & $\mathrm{CS}$ & CS & CS & $\mathrm{CS}$ \\
\hline DMF & $\mathrm{CS}$ & $\mathrm{CS}$ & $\mathrm{CS}$ & $\mathrm{CS}$ & $\mathrm{CS}$ & $\mathrm{CS}$ & $\mathrm{CS}$ & $\mathrm{CS}$ & $\mathrm{CS}$ \\
\hline Water & $\mathrm{N}$ & $\mathrm{P}$ & $\mathrm{P}$ & $\mathrm{P}$ & $\mathrm{N}$ & $\mathrm{P}$ & $\mathrm{N}$ & $\mathrm{N}$ & $\mathrm{N}$ \\
\hline
\end{tabular}

$\mathrm{N}=$ insoluble, $\mathrm{P}=$ partially soluble, $\mathrm{CS}=$ completely soluble, $\mathrm{S} . \mathrm{B}=$ Schiff base

TABLE 3. IR data of Schiff base and its metals complexes

\begin{tabular}{|c|c|c|c|c|c|c|c|c|c|}
\hline \multirow{2}{*}{ Compounds } & \multirow[t]{2}{*}{$v \mathrm{OH}$} & \multirow[t]{2}{*}{$v \mathrm{~N}-\mathrm{H}$} & \multirow[t]{2}{*}{$v \mathrm{C}=\mathrm{N}$} & \multicolumn{2}{|c|}{$v \mathrm{NO}_{2}$} & \multirow[t]{2}{*}{$v$ Ar-O } & \multirow[t]{2}{*}{$v \mathrm{~N}-\mathrm{N}$} & \multirow[t]{2}{*}{$v \mathrm{M}-\mathrm{N}$} & \multirow[t]{2}{*}{$v \mathrm{M}-\mathrm{O}$} \\
\hline & & & & $v_{1}$ & $v_{2}$ & & & & \\
\hline $\mathrm{C}_{13} \mathrm{H}_{10} \mathrm{~N}_{4} \mathrm{O}_{5}$ & $3300-3520$ & 3273 & 1616.79 & 1513.41 & 1334.55 & 1272.00 & 1085.80 & ----- & ----- \\
\hline $\mathrm{Ni}\left(\mathrm{C}_{13} \mathrm{H}_{10} \mathrm{~N}_{4} \mathrm{O}_{5}\right)_{2}$ & ------ & 3275 & 1615.00 & 1513.19 & 1334.00 & 1272.66 & 1144.27 & 660 & 520 \\
\hline $\mathrm{Co}\left(\mathrm{C}_{13} \mathrm{H}_{10} \mathrm{~N}_{4} \mathrm{O}_{5}\right)_{2}$ & ------ & 3270 & 1616.48 & 1514.49 & 1335.07 & 1270.45 & 1144.66 & 663 & 533 \\
\hline $\mathrm{Cu}\left(\mathrm{C}_{13} \mathrm{H}_{10} \mathrm{~N}_{4} \mathrm{O}_{5}\right)_{2}$ & ------- & 3273 & 1616.48 & 1510.10 & 1334.54 & 1260.73 & 1056.99 & 670 & 524 \\
\hline $\mathrm{Zn}\left(\mathrm{C}_{13} \mathrm{H}_{10} \mathrm{~N}_{4} \mathrm{O}_{5}\right)_{2}$ & ------- & 3272 & 1616.33 & 1513.66 & 1334.45 & 1268.00 & 1135.23 & 688 & 545 \\
\hline $\mathrm{Mn}\left(\mathrm{C}_{13} \mathrm{H}_{10} \mathrm{~N}_{4} \mathrm{O}_{5}\right)_{2}$ & ------- & 3265 & 1615.00 & 1513.66 & 1334.00 & 1272.66 & 1144.27 & 673 & 528 \\
\hline $\mathrm{Cd}\left(\mathrm{C}_{13} \mathrm{H}_{10} \mathrm{~N}_{4} \mathrm{O}_{5}\right)_{2}$ & ------- & 3269 & 1615.21 & 1509.21 & 1332.75 & 1270.05 & 113368 & 677 & 543 \\
\hline $\mathrm{V}\left(\mathrm{C}_{13} \mathrm{H}_{10} \mathrm{~N}_{4} \mathrm{O}_{5}\right)_{2}$ & ------- & 3267 & 1615.55 & 1511.69 & 1333.17 & 1272.13 & 1134.74 & 681 & 547 \\
\hline $\mathrm{Au}\left(\mathrm{C}_{13} \mathrm{H}_{10} \mathrm{~N}_{4} \mathrm{O}_{5}\right)_{3}$ & ------- & 3260 & 1615.97 & 1513.22 & 1334.02 & 1272.64 & 1144.68 & 678 & 540 \\
\hline
\end{tabular}

octahedral. The Schiff base is bidentate. Each metal ion coordinating to the two $\mathrm{C}=\mathrm{N}$ azomethine moieties and two oxygen atoms from each hydroxyl group of phenol to completed the octahedral structure.

\section{ELECTRONIC SPECTROSCOPY}

The ultraviolet absorption spectrums of the prepared compounds took in DMSO at $25^{\circ} \mathrm{C}$. The data of the maximum absorption wavelength $(\lambda \max )$ are given in 
Table 4. The characteristic bands of SDNPH Schiff base assigned at 275,296 and $405 \mathrm{~nm}$, respectively. The higher energy band represent electron transition $\pi \rightarrow \pi^{*}$ in benzene sphere and imine or azomethene moiety (Felico et al. 2001; Guo et al. 2006). The lower energy transition represents the $\mathrm{n} \rightarrow \pi^{*}$ transition of $\mathrm{N}$ atom $\mathrm{p}$-orbital lone pair in imine and benzene sphere (Youssef et al. 2008). The ultraviolet absorption spectrums of all the coordination compounds Ln (II) and Ln (III) Schiff base metals complexes show resemblance which represents alike structures are given in the Table 4. The difference between the spectrums of the complexes and ligand after complexation confirmed coordination bond. Further changing in the wavelength also indicated that the coordination of the schiff base occurred to the metals ions

\section{ANTIBACTERIAL STUDY}

The antibacterial activity was evaluated by in vitro method against four bacteria strains, gram positive $B$. subtilis and $S$. aureus and gram negative $E$. coli and $S$. typhi bacteria. The two concentrations ( 1 and $2 \mathrm{mg} / \mathrm{mL}$ ) of each sample were used against tested microorganism through agar well diffused assay. In well diffused method, diffusion of microorganism occurred on nutrient agar medium plates and incubated for $24 \mathrm{~h}$ at $37^{\circ} \mathrm{C}$. The inhibitory zone of microorganism growth around the well was measured in millimeter. The data of the antibacterial activity of all the compounds are given in Table 5. All the metals complexes showed high activity than Schiff base, it is due to the coordination of Schiff base with metals which enhance their activities significantly (Chohan et al. 2004). Among the metals complexes Copper complex showed high activity against $E$. coli and $S$. typhi. Zinc complex showed activity against $B$. subtilis. The tetracycline was used as standard for E. coli, S. typhi and cephradine used for B. subtilis and S. aureus.

\section{ANTIFUNGAL ACTIVITY}

Antifungal study was evaluated against four fungus species, C. albicans, $R$. stolonifer, $T$. viride and A. nigar, in their in vitro method through agar well diffused standard protocol.
For this purpose, potato dextrose agar was used as medium. In this method, each species of fungus was streaked on potato dextrose agar medium plates and incubated for 48 $\mathrm{h}$ at $37^{\circ} \mathrm{C}$. Two different concentrations of 1 and $2 \mathrm{mg} /$ $\mathrm{mL}$ were applied. The activity was determined by the calculation of zones of inhibition in $(\mathrm{mm})$ are tabulated in the Table 6. Schiff base showed less antifungal activity, while the metals complexes showed high activities. The experimental data was evaluated with standard antifungal medicine fluconazole under similar condition followed in tested compounds.

Copper complex showed high activity among the metals complexes against $C$. albicans followed by $R$. stolonifer, T. viride and A. nigar. Nickel complex also showed high activity toward $C$. albicans and $R$. stolonifer than other meal complexes.

The results showed that some metals complexes show high activities as compared of Schiff base, however, it is also known that Schiff base containing $\mathrm{N}$ and $\mathrm{O}$ donor atoms increased its activity through complexation with metals ions (Arulpriya et al. 2010; Chohan et al. 2002).

\section{ANTI-OXIDANT STUDY}

The DPPH free radical protocol was employed to study the standard free radical potentials. The antioxidant activity of Schiff base salicylalde-2, 4-dinitrophenylhydrazone and its metals complexes were analyzed by in-vitro method. Different concentrations $(10,20,30,40 \& 50 \mathrm{ppm})$ of tested compounds were used for the evaluation of antioxidant activity. Ascorbic acid was used as a standard which show $100 \%$ activity. The scavenging reaction between (DPPH) free radical and an antioxidant (H-D) can be written as:

$$
\begin{array}{cc}
(\mathrm{DPPH})+(\mathrm{H}-\mathrm{D}) & \rightarrow \text { DPPH-H }+(\mathrm{D}) \\
(\text { Purple }) & (\text { Yellow })
\end{array}
$$

The results of free radical scavenging activities of $\mathrm{Cu}$, $\mathrm{Ni}, \mathrm{Cd}$, and $\mathrm{Au}$ Complexes and $\mathrm{V}, \mathrm{Mn}, \mathrm{Co}, \mathrm{Zn}$ are given in the Figures 1 and 2, respectively. Ligand showed less activity while on complexation with metals increased the

TABLE 4. UV-visible spectral data wavelength (nm) for the Schiff base and its complexes

\begin{tabular}{clcl}
\hline S. No & \multicolumn{1}{c}{ Compounds } & Wavelength $(\mathrm{nm})$ & Band assignment \\
\hline 1 & $\mathrm{C}_{13} \mathrm{H}_{10} \mathrm{~N}_{4} \mathrm{O}_{5}$ & $275,296,405$ & $\pi \rightarrow \pi^{*}, \mathrm{n} \rightarrow \pi^{*}$ \\
2 & $\mathrm{Ni}\left(\mathrm{C}_{13} \mathrm{H}_{10} \mathrm{~N}_{4} \mathrm{O}_{5}\right)_{2}$ & $339,350,470$ & $\pi \rightarrow \pi^{*}, \mathrm{n} \rightarrow \pi^{*}$ \\
3 & $\mathrm{Co}\left(\mathrm{C}_{13} \mathrm{H}_{10} \mathrm{~N}_{4} \mathrm{O}_{5}\right)_{2}$ & $297,315,460$ & $\pi \rightarrow \pi^{*}, \mathrm{n} \rightarrow \pi^{*}$ \\
4 & $\mathrm{Cu}\left(\mathrm{C}_{13} \mathrm{H}_{10} \mathrm{~N}_{4} \mathrm{O}_{5}\right)_{2}$ & $312,319,465$ & $\pi \rightarrow \pi^{*}, \mathrm{n} \rightarrow \pi^{*}$ \\
5 & $\mathrm{Zn}\left(\mathrm{C}_{13} \mathrm{H}_{10} \mathrm{~N}_{4} \mathrm{O}_{5}\right)_{2}$ & $325,339,474$ & $\pi \rightarrow \pi^{*}, \mathrm{n} \rightarrow \pi^{*}$ \\
6 & $\mathrm{Mn}\left(\mathrm{C}_{13} \mathrm{H}_{10} \mathrm{~N}_{4} \mathrm{O}_{5}\right)_{2}$ & $343,360,500$ & $\pi \rightarrow \pi^{*}, \mathrm{n} \rightarrow \pi^{*}$ \\
7 & $\mathrm{Cd}\left(\mathrm{C}_{13} \mathrm{H}_{10} \mathrm{~N}_{4} \mathrm{O}_{5}\right)_{2}$ & $300,360,484$ & $\pi \rightarrow \pi^{*}, \mathrm{n} \rightarrow \pi^{*}$ \\
8 & $\mathrm{~V}\left(\mathrm{C}_{13} \mathrm{H}_{10} \mathrm{~N}_{4} \mathrm{O}_{5}\right)_{2}$ & $285,304,495$ & $\pi \rightarrow \pi^{*}, \mathrm{n} \rightarrow \pi^{*}$ \\
9 & $\mathrm{Au}\left(\mathrm{C}_{13} \mathrm{H}_{10} \mathrm{~N}_{4} \mathrm{O}_{5}\right)_{3}$ & $291,331,447$ & $\pi \rightarrow \pi^{*}, \mathrm{n} \rightarrow \pi^{*}$ \\
\hline
\end{tabular}


TABLE 5. Zone of inhibition for antibacterial study

\begin{tabular}{|c|c|c|c|c|c|c|c|c|}
\hline \multirow{3}{*}{ Compounds } & \multicolumn{4}{|c|}{ Gram positive } & \multicolumn{4}{|c|}{ Gram negative } \\
\hline & \multicolumn{2}{|c|}{ B. subtilis } & \multicolumn{2}{|c|}{ S. aureus } & \multicolumn{2}{|c|}{ E. coli } & \multicolumn{2}{|c|}{ S. thypi } \\
\hline & \multicolumn{2}{|c|}{$1 \mathrm{mg} / \mathrm{mL} 2 \mathrm{mg} / \mathrm{mL}$} & \multicolumn{2}{|c|}{$1 \mathrm{mg} / \mathrm{mL} 2 \mathrm{mg} / \mathrm{mL}$} & \multicolumn{2}{|c|}{$1 \mathrm{mg} / \mathrm{mL} 2 \mathrm{mg} / \mathrm{mL}$} & \multicolumn{2}{|c|}{$1 \mathrm{mg} / \mathrm{mL} 2 \mathrm{mg} / \mathrm{mL}$} \\
\hline $\mathrm{C}_{13} \mathrm{H}_{10} \mathrm{~N}_{4} \mathrm{O}_{5}$ & 07 & 08 & 00 & 00 & 00 & 00 & 00 & 00 \\
\hline $\mathrm{Cu}\left(\mathrm{C}_{13} \mathrm{H}_{10} \mathrm{~N}_{4} \mathrm{O}_{5}\right)_{2}$ & 12 & 16 & 11 & 15 & 8 & 12 & 08 & 10 \\
\hline $\mathrm{Ni}\left(\mathrm{C}_{13} \mathrm{H}_{10} \mathrm{~N}_{4} \mathrm{O}_{5}\right)_{2}$ & 08 & 10 & 10 & 12 & 07 & 09 & 06 & 09 \\
\hline $\mathrm{Zn}\left(\mathrm{C}_{13} \mathrm{H}_{10} \mathrm{~N}_{4} \mathrm{O}_{5}\right)_{2}$ & 09 & 11 & 11 & 13 & 10 & 12 & 07 & 09 \\
\hline $\mathrm{Co}\left(\mathrm{C}_{13} \mathrm{H}_{10} \mathrm{~N}_{4} \mathrm{O}_{5}\right)_{2}$ & 00 & 06 & 07 & 10 & 00 & 00 & 00 & 00 \\
\hline $\mathrm{Mn}\left(\mathrm{C}_{13} \mathrm{H}_{10} \mathrm{~N}_{4} \mathrm{O}_{5}\right)_{2}$ & 00 & 06 & 08 & 10 & 06 & 07 & 05 & 06 \\
\hline $\mathrm{Au}\left(\mathrm{C}_{13} \mathrm{H}_{10} \mathrm{~N}_{4} \mathrm{O}_{5}\right)_{3}$ & 06 & 09 & 07 & 09 & 00 & 00 & 06 & 00 \\
\hline $\mathrm{Cd}\left(\mathrm{C}_{13} \mathrm{H}_{10} \mathrm{~N}_{4} \mathrm{O}_{5}\right)_{2}$ & 06 & 08 & 07 & 09 & 00 & 00 & 00 & 00 \\
\hline $\mathrm{V}\left(\mathrm{C}_{13} \mathrm{H}_{10} \mathrm{~N}_{4} \mathrm{O}_{5}\right)_{2}$ & 00 & 06 & 00 & 07 & 00 & 00 & 00 & 00 \\
\hline Tetracycline & 20 & 22 & 20 & 22 & ---- & ---- & --- & --- \\
\hline Cephradine & ----- & ---- & ---- & ----- & 16 & 18 & 15 & 17 \\
\hline
\end{tabular}

TABLE 6. Zone of inhibition for anti-fungus study

\begin{tabular}{lcccccccc}
\hline \multirow{2}{*}{ Compounds } & \multicolumn{2}{c}{ C. albicans } & \multicolumn{2}{c}{ R. stolonifer } & \multicolumn{2}{c}{ T. viride } & \multicolumn{2}{c}{ A. nigar } \\
\cline { 2 - 8 } & $1 \mathrm{mg} / \mathrm{mL} 2 \mathrm{mg} / \mathrm{mL}$ & $1 \mathrm{mg} / \mathrm{mL} 2$ & $\mathrm{mg} / \mathrm{mL}$ & $1 \mathrm{mg} / \mathrm{mL} 2$ & $\mathrm{mg} / \mathrm{mL}$ & $1 \mathrm{mg} / \mathrm{mL} 2 \mathrm{mg} / \mathrm{mL}$ \\
\hline $\mathrm{C}_{13} \mathrm{H}_{10} \mathrm{~N}_{4} \mathrm{O}_{5}$ & 00 & 00 & 00 & 00 & 00 & 00 & 00 & 00 \\
$\mathrm{Cu}\left(\mathrm{C}_{13} \mathrm{H}_{10} \mathrm{~N}_{4} \mathrm{O}_{5}\right)_{2}$ & 14 & 16 & 12 & 14 & 11 & 12 & 10 & 11 \\
$\mathrm{Zn}\left(\mathrm{C}_{13} \mathrm{H}_{10} \mathrm{~N}_{4} \mathrm{O}_{5}\right)_{2}$ & 08 & 10 & 07 & 19 & 08 & 09 & 06 & 08 \\
$\mathrm{Ni}_{\left(\mathrm{C}_{13} \mathrm{H}_{10} \mathrm{~N}_{4} \mathrm{O}_{5}\right)_{2}}$ & 12 & 13 & 10 & 12 & 07 & 09 & 06 & 08 \\
$\mathrm{Co}\left(\mathrm{C}_{13} \mathrm{H}_{10} \mathrm{~N}_{4} \mathrm{O}_{5}\right)_{2}$ & 08 & 09 & 07 & 09 & 06 & 08 & 00 & 00 \\
$\mathrm{Mn}\left(\mathrm{C}_{13} \mathrm{H}_{10} \mathrm{~N}_{4} \mathrm{O}_{5}\right)_{2}$ & 08 & 10 & 06 & 08 & 07 & 09 & 06 & 07 \\
$\mathrm{Au}\left(\mathrm{C}_{13} \mathrm{H}_{10} \mathrm{~N}_{4} \mathrm{O}_{5}\right)_{2}$ & 06 & 08 & 06 & 09 & 06 & 07 & 05 & 06 \\
$\mathrm{Cd}\left(\mathrm{C}_{13} \mathrm{H}_{10} \mathrm{~N}_{4} \mathrm{O}_{5}\right)_{2}$ & 06 & 07 & 05 & 06 & 00 & 06 & 00 & 05 \\
$\mathrm{~V}\left(\mathrm{C}_{13} \mathrm{H}_{10} \mathrm{~N}_{4} \mathrm{O}_{5}\right)_{2}$ & 05 & 06 & 00 & 06 & 06 & 07 & 06 & 06 \\
Fluconazole & 19 & 22 & 17 & 20 & 16 & 20 & 15 & 20 \\
\hline
\end{tabular}

activity significantly. All the metal complexes showed comparable or less activity than that of standard (Ascorbic acid). The high antioxidant potential of these complexes can be recognized to the electron retreating consequences of the $\mathrm{Cu}(\mathrm{II}), \mathrm{Ni}(\mathrm{II}), \mathrm{Zin}(\mathrm{II})$ and $\mathrm{Cd}(\mathrm{II})$ ions which assisted the liberate of hydrogen to squeeze the DPPH free radical (Tetteh et al. 2014).

\section{CYTOTOXICITY}

Cytotoxicity was evaluated through brine shrimp lethality assay. Different concentrations $(1000,250 \& 100 \mu \mathrm{g} / \mathrm{mL})$ of each sample were tested against 10 naupuli in vial. Blank solution of DMSO was used as negative control and doxorubicin $4 \mathrm{mg} / \mathrm{mL}$ was used as positive control. After the completion of incubation period, dead naupli were counted in each vial. The results found were promising given in the Table 7. Metals complexes showed high percent mortality as compared to ligand, respectively (Tarafder et al. 2000). These finding matched with the previous studied showed that the metals, complexes of Copper, Nickel, Zinc and Gold showed 100\% mortality at all concentrations (Afrasiabi et al. 2005).

\section{CONCLUSION}

The results showed that the synthesized compounds showed significant antimicrobial (antibacterial and antifungal), antioxidant and cytotoxic activities. Schiff base showed less biologicals activities than metals complexes. Among the metals complexes Cu (II), Zinc (II), Co (II) and $\mathrm{Ni}$ (II) were found to show high activities than other metals complexes. 


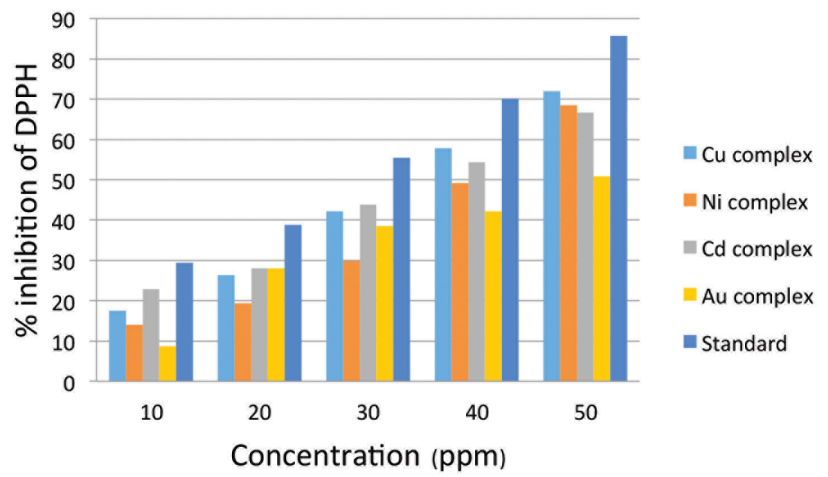

FIGURE 1. Antioxidant potential of complexes of $\mathrm{Cu}, \mathrm{Ni}, \mathrm{Cd}, \mathrm{Au}$ and standard

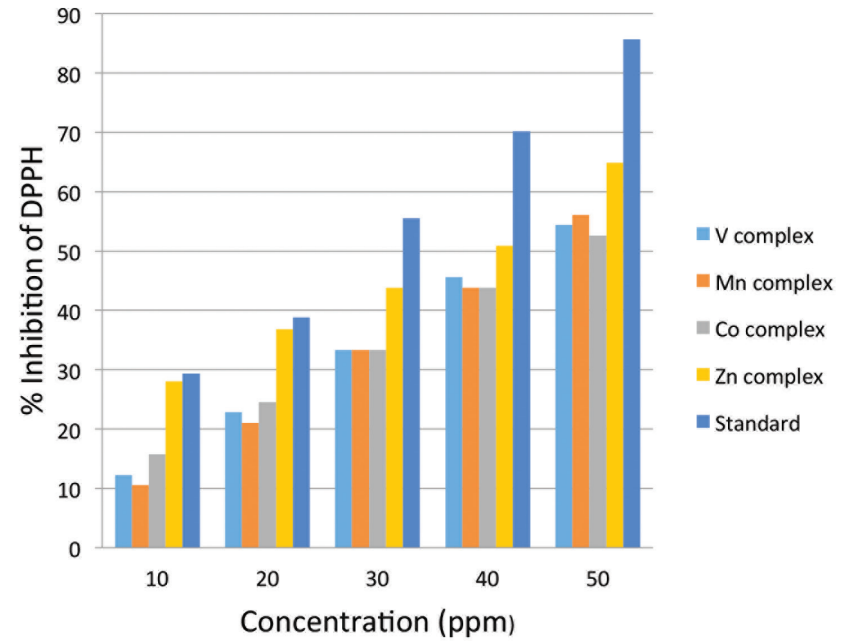

FIGURE 2. Antioxidant potential of complexes of $\mathrm{V}, \mathrm{Mn}$, $\mathrm{Co}, \mathrm{Zn}$ and standard

TABLE 7. \% Mortality of Schiff base and its complexes

\begin{tabular}{lccc}
\hline Compounds & \multicolumn{3}{c}{$\%$ Mortality } \\
\cline { 2 - 4 } & $100 \mu \mathrm{g} / \mathrm{mL}$ & $250 \mu \mathrm{g} / \mathrm{mL}$ & $1000 \mu \mathrm{g} / \mathrm{mL}$ \\
\hline $\mathrm{C}_{13} \mathrm{H}_{10} \mathrm{~N}_{4} \mathrm{O}_{5}$ & 50 & 50 & 60 \\
$\mathrm{Cu}\left(\mathrm{C}_{13} \mathrm{H}_{10} \mathrm{~N}_{4} \mathrm{O}_{5}\right)_{2}$ & 100 & 100 & 100 \\
$\mathrm{Ni}\left(\mathrm{C}_{13} \mathrm{H}_{10} \mathrm{~N}_{4} \mathrm{O}_{5}\right)_{2}$ & 100 & 100 & 100 \\
$\mathrm{Zn}\left(\mathrm{C}_{13} \mathrm{H}_{10} \mathrm{~N}_{4} \mathrm{O}_{5}\right)_{2}$ & 100 & 100 & 100 \\
$\mathrm{Co}\left(\mathrm{C}_{13} \mathrm{H}_{10} \mathrm{~N}_{4} \mathrm{O}_{5}\right)_{2}$ & 70 & 70 & 80 \\
$\mathrm{Mn}\left(\mathrm{C}_{13} \mathrm{H}_{10} \mathrm{~N}_{4} \mathrm{O}_{5}\right)_{2}$ & 70 & 70 & 80 \\
$\mathrm{Au}\left(\mathrm{C}_{13} \mathrm{H}_{10} \mathrm{~N}_{4} \mathrm{O}_{5}\right)_{3}$ & 100 & 100 & 100 \\
$\mathrm{Cd}\left(\mathrm{C}_{13} \mathrm{H}_{10} \mathrm{~N}_{4} \mathrm{O}_{5}\right)_{2}$ & 60 & 60 & 80 \\
$\mathrm{~V}\left(\mathrm{C}_{13} \mathrm{H}_{10} \mathrm{~N}_{4} \mathrm{O}_{5}\right)_{2}$ & 40 & 40 & 60 \\
\hline
\end{tabular}

\section{ACKNOWLEDGEMENTS}

The authors extend their appreciation to the Deanship of Scientific Research at King Saud University for funding this work through research group no (RG-1440-100).

\section{REFERENCES}

Afrasiabi, Z., Sinn, E., Lin, W., Ma, Y., Campana, C. \& Padhye, S. 2005. Nickel (II) complexes of naphthaquinonethiosemicarbazone and semicarbazone synthesis, structure, spectroscopy and biological activity. Journal of Inorganic Biochemistry 9: 1526-1530. 
Amrata, P., Agrawal, M.C. \& Bhatnagar, R.K. 2014. Synthesis, spectral characterization and antifungal activity of a new Schiff base hydrazone derived from 2, 4-dinitrophenyl hydrazine. International Journal of Chemical and Pharmaceutical Sciences 5(1): 456-461.

Arulpriya, P., Lalitha, P. \& Hemalatha, S. 2010. In vitro antioxidant testing of the extracts of Samaneasaman (Jacq.) Merr. Der chemica Sinica 1: 73-79.

Chohan, Z.H., Supuran, C.T. \& Scozzafava, A. 2004. Metalloantibiotics: Synthesis and antibacterial activity of cobalt(II), copper(II), nickel(II) and zinc(II) complexes of kefzol. Journal of Enzyme Inhibition and Medicinal Chemistry 19(1): 79-84.

Chohan, Z.H., Scozzafava, A. \& Supuran, C.T. 2002. Unsymmetrical 1, 1- disubstituted ferrocenes: Synthesis of $\mathrm{Co}(\mathrm{ii}), \mathrm{Cu}$ (ii), $\mathrm{Ni}$ (ii) and $\mathrm{Zn}$ (ii) chelates of ferrocenyl -1-thiadiazolo-1-tetrazole, -1-thiadiazolo-1-triazole and -1-tetrazolo-1-triazole with antimicrobial properties. $J$. Enzym. Inhib. Med. Chem. 17: 261-266.

Felico, R.C., Canalheiro, E.T.G. \& Dockal, E.R. 2001. Preparation, characterization and thermogravimetric studies of [N, N-cis-1, 2-cyclohexylene bis(salicylideneaminato)] cobalt(II) and [N, $\mathrm{N}$-( \pm -trans-1, 2-cyclo-hexylene bis(salicylideneaminato)] cobalt(II). Polyhedron 20: 261-268.

Florian, A., Uwe, K., Mathias, N. \&Von, K.Z. 2006. 1,2-Diaza3-phospha-cyclopent-5-enen, -penta-3,5-dienen, 1,5-Diaza2,6-diphospha-bicyclo [3.3.0]octa-3,7-dien und einem Cyclohexaphosphan. Z. Naturforsch 61(b): 225-236.

Guo, L., Wu, S., Zeng, F. \& Zhao, J. 2006. in situ synthesis of copper nanoparticles and poly (o-toluidine): A metal-polymer composite material. European Polymer Journal 42: 670-675.

Hajar, S., Shahriare, G., Kheyrollah, M. \& Farshid, S. 2010. Novel mercury(II) complexes of Schiff base ligands: Synthesis and spectral characterization. Der Chemica Sinica 1(1): 39-44.

Hany, S.I., Soha, R., Abdelhadi. \& Hatem, A.A. 2015. Hydrolysis and hydrazinolysis of isatin-based ald- and ketazines. Journal of Chemistry 2015: 1-6.

Hassan, H.M., Omid, P. \& Christoph, J. 2007. Synthesis and spectral characterization of hydrazone schiff bases derived from 2,4-dinitrophenylhydrazine. crystal structure of salicylaldehyde-2,4-dinitrophenylhydrazone. Z. Naturforsch 62b: 717-720.

Jin, L., Chen, J., Song, B., Chen, Z., Yang, Q.S., Hu, D. $\& \mathrm{Xu}$, R. 2006. Synthesis, structure, and bioactivity of $\mathrm{N}^{\prime}$-substitutedbenzylidene-3, 4, 5-trimethoxy benzohydrazide and 3-acetyl-2-substituted phenyl-5-(3, 4, 5-trimethoxyphenyl)-2, 3-dihydro-1, 3, 4-oxadiazole derivatives. Bioorganic \& Medicinal Chemistry Letters 16: 5036-5040.

Kaczmarek, S.M. \& Leniec, G. 2009. EPR and IR investigations of some chromium (III) phosphate (V) compounds. Journal of Non-Crystalline Solids 355: 1289-1438.

Katyal, M. \& Dutt, Y. 1975. Analytical applications of hydrazones. Talanta 22: 151-166.

Leniec, G., Kaczmarek, S.M., Typek, J., Koodziej, B., Grech, E. \& Schilf, W. 2006. Spectroscopic and magnetic properties of gadolinium macrobicycliccryptate complex. J. Phys. Condens. Matter. 18: 9871-9880.

Li-Fei, Z., Xiu-Yun, Y., Dong-Xia, W., Ying, G., Yuan, W. \& YunHui, L. 2012. Synthesis and crystal structures of nickel(ii) and cobalt(ii) complexes with 3-methoxysalicylaldehyde benzoylhydrazide. Asian Journal of Chemistry 24(7): 29092912.
Misbah, U.R., Muhammad, I. \& Muhammad, A. 2013. Synthesis, characterization and in vitro antimicrobial studies of schiffbases derived from acetyl acetone and amino acids and their oxovanadium(iv) complexes. American Journal of Applied Chemistry 1(4): 59-66.

Ochiai, E. 1977. Bioinorganic Chemistry: An Introduction Boston: Allyn and Bacon. p. 468.

Rajarajan, M., Senbagam, R., Vijayakumar, R., Balaji, S., Manikandan, V., Vanangamudi, G. \& Thirunarayanan, G. 2016. Synthesis, spectral correlations and antimicrobial activities of substituted 4-((E)-2-benzylidenehydrazinyl) benzonitriles. Indian Journal of Chemistry 55B: 197-206.

Savini, L., Chiasserini, L., Gaeta,A.\& Pellerano, C. 2002. Synthesis and anti-tubercular evaluation of 4-quinolylhydrazones. Biorganic and Medical Chemistry 10(7): 2193-2198.

Shahawi, M.S.E., Jahdali, M.S.A., Bashammakh, A.S., Sibaai, A.A.A. \& Nassef, H.M. 2013. Spectroscopic and electrochemical characterization of some Schiff base metal complexes containing benzoin moiety. Spectrochim. Acta A 113: 459-465.

Silverstein, R.M., Bassler, G.C. \& Movril, T.C. 1981. Spectroscopic Identification of Organic Compounds. 4th ed. New York: Wiley.

Tarafder, M.T.H., Ali, M.A., Wee, D.J., Azahari, K., Silong, S. \& Crouse, K.A. 2000. Complexes of a tridentate ONS Schiff base. Synthesis and biological properties. Trans. Met. Chem 25(4): 456-460

Tetteh, S., Dodoo, D.K., Appiah, O.R. \& Tuffour, I. 2014. Spectroscopic characterization in vitro cytotoxicity and antioxidant activity of mixed ligand palladium (II) chloride complexes bearing nucleobases. J. Inorg. Chem. 4(5): 670676.

Youssef, T.A. 2008. Reactions of chromium and molybdenum carbonyls with bis-(salicylaldehyde)-1, 3-propylenediimine Schiff base. J. Coord. Chem 61: 816-819.

Zahid, H., Chohan, S. \& Sherazi, K.A. 1997. Biological role of cobalt (ii), copper (ii) and nickel (ii) metal ions on the antibacterial properties of some nicotinoyl-hydrazine derived compounds. Metal Based Drugs 4(2): 69-74.

Zeyrek, C.T., Elmali, A. \& Elerman, Y. 2006. Super-exchange interaction in a chair-piperazine bridged dicopper(II/II) complex: Synthesis, crystal structure, magnetic properties and molecular orbital calculations. Z. Naturforsch 61(b): 237-242.

Iqbal Hussain, Aaqib Ullah \& Atta Ullah Khan

Department of Chemistry

Islamia College University Peshawar

Pakistan

Wali Ullah Khan

Department of Chemistry

Abdul Wali Khan University Mardan

Pakistan

Riaz Ullah* \& Abdelaaty A. Shahat Almoqbil A. Naser

Department of Pharmocognosy (Medicinal, Aromatic and Poisonous Plants Research Center)

College of Pharmacy

King Saud University, Riyadh

Saudi Arabia 
Abdelaaty A. Shahat Phytochemistry Department

National Research Centre

P.O. Box 1262233

El Bohouth St., Dokki, Giza

Egypt

Hafiz Majid Mahmood

Department of Pharmacology

College of Pharmacy

King Saud University

P.O. Box 2457, Riyadh 11451

Saudi Arabia
*Corresponding author; email: rullah@ksu.edu.sa

Received: 15 January 2019

Accepted: 24 April 2019 